\title{
Delineation of a paravalvular leak origin between a rigid mitral ring and supra-annular implanted mechanical mitral prosthetic valve
}

\begin{abstract}
Paravalvular leakage is not a rare condition among patients with mitral prostheses. Two-dimensional echocardiographic approaches are not sufficient to determine the origin of paravalvular leak that occurs after prosthetic mitral valve replacement. Real-time three-dimensional transesophageal echocardiography provides detailed structural identification of paravalvular leak origin and defect morphology compared to two-dimensional transesophageal echocardiography. Here we present a patient with paravalvular mitral regurgitation from a slit like defect between the mitral anular ring and the prosthetic valve delineated with the utility of $3 \mathrm{D}$ full volume and $3 \mathrm{D}$ color flow imaging modalities.
\end{abstract}

Keywords: mitral ring, paravalvular leak, transesophageal, echocardiography
Volume I Issue 2 - 2014

\author{
Macit Kalcik,' Mahmut Yesin,' M Ozan \\ Gursoy,' Mehmet Ozkan² \\ 'Department of Cardiology, Kosuyolu Kartal Heart Training and \\ Research Hospital,Turkey \\ ${ }^{2}$ Department of Cardiology, Kars Kafkas University, Turkey
}

Correspondence: Macit Kalcik, Department of Cardiology, Kosuyolu Kartal Heart Training and Research Hospital, Esentepe Mah, Milangaz Caddesi Unluer Sitesi B-Blok, No:22, Kartal/ Istanbul,Turkey, Tel 90-536-4921789, Emailmacitkalcik@yahoo.com
Abbreviations: TEE, Transesophageal echocardiography; 2D, two dimensional; 3D, 3 dimensional

\section{Case report}

A 65year-old woman suffering from severe mitral valve regurgitation underwent mitral ring annuloplasty (31 no Saint Jude Medical Rigid Ring). After weaning from cardiopulmonary bypass pump, intraoperative transesophageal echocardiography (TEE) was performed this revealed withstanding severe mitral regurgitation. Therefore a supraannular 28 no bileaflet ATS-AP mechanical mitral valve was implanted over the mitral ring and intraoperative TEE showed normally functioning mitral prosthesis. The surgery was completed without complication. The patient experienced an uneventful postoperative period but was readmitted to hospital with ongoing NYHA Class 2 dyspnea one month after discharge. Transthoraic echocardiogram did not show any pericardial effusion but a moderate mitral regurgitation jet detected by color Doppler imaging (Figure 1A). TEE was performed to depict the origin of the regurgitant jet. Two dimensional (2D) TEE showed a moderate paravalvular leakage with the help of color Doppler imaging (Figure 1B). Subsequently, real-time 3 dimensional (3D) TEE revealed a regurgitates jet arising from a slit like defect between the mitral anular ring and the prosthetic valve with the utility of $3 \mathrm{D}$ full-volume and $3 \mathrm{D}$ color flow imaging modalities (Figures $2 \mathrm{~A} \& 2 \mathrm{~B}$ ).
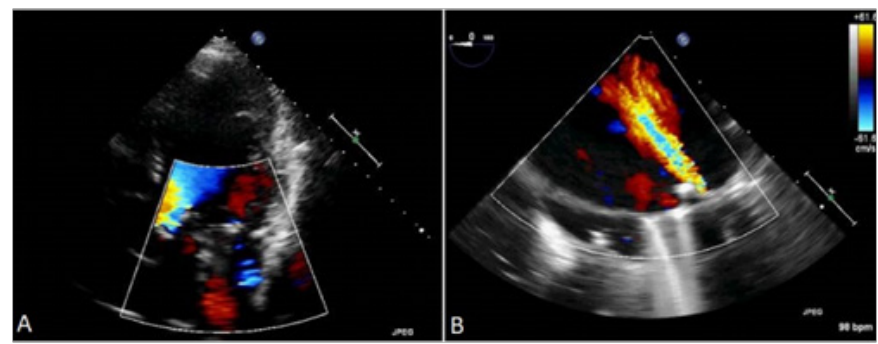

Figure I Transthorasic echocardiogram on apical five chamber view (A) and two dimensional transesophageal echocardiogram (B) showing moderate paravalvular mitral regurgitation by color Doppler imaging.

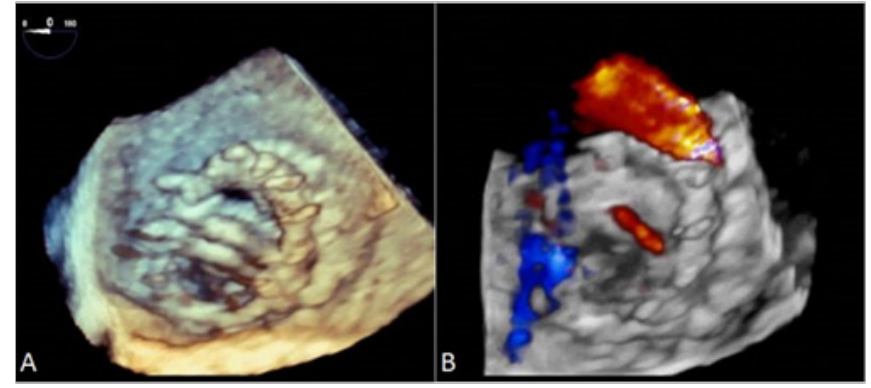

Figure 2 Full volume real-time three-dimensional transesophagea echocardiography revealing supra annulary implanted mitral mechanical prosthesis on a rigid mitral ring $(A)$ and identification of paravalvular leak origin with the utility of $3 \mathrm{D}$ color flow imaging modality $(\mathrm{B})$.

Paravalvular leakage is not a rare condition among patients with mitral prostheses ${ }^{1}$ but a leakage between a mitral ring and mechanic prosthetic valve has not been reported so far. Real-time 3D TEE provides detailed structural identification of paravalvular leak origin and defect morphology compared to 2D TEE. ${ }^{2-5}$

\section{Acknowledgments}

None.

\section{Conflicts of interest}

Authors declare that there are no conflicts of interest.

\section{References}

1. Gursoy OM, Astarcioglu MA, Gokdeniz T, et al. Severe mitral paravalvular leakage: echo-morphologic description of 47 patients from real-time three-dimensional transesophageal echocardiography perspective. Anatolian Journal of Cardiology. 2013;13(7):633-640.

2. Kronzon I, Sugeng L, Perk G, et al. Real-time 3-dimensional transesophageal echocardiography in the evaluation of post-operative mitral annuloplasty ring and prosthetic valve dehiscence. $J$ Am Coll Cardiol. 2009;53(17):1543-1547. 
3. Singh P, Manda J, Hsiung MC, et al. Live/real time three-dimensional transesophageal echocardiographic evaluation of mitral and aortic valve prosthetic paravalvular regurgitation. Echocardiography 2009;26(8):980-987.

4. Biner S, Kar S, Siegel RJ, et al. Value of color Doppler three-dimensional transesophageal echocardiography in the percutaneous closure of mitral prosthesis paravalvular leak. Am J Cardiol. 2010;105(7):984-989.
5. Yildiz M, Duran NE, Gokdeniz T, et al. The value of real-time threedimensional transesophageal echocardiography in the assessment of paravalvular leak origin following prosthetic mitral valve replacement. Ars Turk Soc Cardiol. 2009;37(6):371-377. 